\title{
Minimally Invasive Excision of Epidermal Cysts through a Small Hole Made by a $\mathrm{CO}_{2}$ Laser
}

\author{
Seung Wook Song, Jin Sik Burm, Won Yong Yang, Sang Yoon Kang \\ Department of Plastic and Reconstructive Surgery, Kyung Hee University School of Medicine, Seoul, Korea
}

To improve the cosmetic results of removing epidermal cysts, minimally invasive methods have been proposed. We proposed a new minimally invasive method that completely removes a cyst through a small hole made by a $\mathrm{CO}_{2}$ laser. Twenty-five patients with epidermal cysts, which were 0.5 to $1.5 \mathrm{~cm}$ in diameter, non-inflamed, and freely movable, were treated. All of the patients were satisfied with the cosmetic results. This method is simple and results in minimal scarring and low recurrence rates without complications.

Keywords Epidermal cyst / Lasers

\author{
Correspondence: Jin Sik Burm \\ Department of Plastic and \\ Reconstructive Surgery, \\ Kyung Hee University School of \\ Medicine, 23 Kyungheedae-ro, \\ Dongdaemun-gu, Seoul 130-872, \\ Korea \\ Tel: +82-2-958-8431 \\ Fax: +82-2-963-5638 \\ E-mail: jsburm@gmail.com
}

This article was presented as a poster at the 69th Congress of the Korean Society of Plastic and Reconstructive Surgeons on November 11-13, 2011 in Seoul, Korea.

No potential conflict of interest relevant to this article was reported.

Received: 11 Jul $2013 \bullet$ Revised: 20 Sep $2013 \bullet$ Accepted: 21 Sep 2013

pISSN: 2234-6163・ elSSN: 2234-6171 • http://dx.doi.org/10.5999/aps.2014.41.1.85• Arch Plast Surg 2014;41:85-88

\section{INTRODUCTION}

Epidermal cysts are one of the most common benign epithelial tumors. They are firm-to-fluctuant, slow-growing, dome-shaped lesions that frequently appear on the trunk, neck, face, scrotum, or behind the ears [1]. Surgical excision is commonly used for treating these lesions with the inevitable sequelae of scarring [25]. Anatomically, the apex of an epidermal cyst is attached to the dermis of the skin. However, the remaining portion of the cyst underneath the skin loosely attaches to the subcutaneous tissue and is freely mobile over it. Recently, on this anatomical basis, minimally invasive methods have been suggested to avoid the long scar resulting from conventional surgical excision. We designed a new minimally invasive method that completely removes the cyst through a small hole made by a $\mathrm{CO}_{2}$ laser.

\section{IDEA}

The subjects were 25 patients ( 15 males and 10 females; aged, 18 to 67 years) with epidermal cysts. A total of 25 cysts included 16 on the face, 6 on the neck, 1 on the upper chest, and 2 on the sole of the foot. The inclusion criteria were the following: being $0.5 \mathrm{~cm}$ to $1.5 \mathrm{~cm}$ in diameter, non-inflamed, and freely movable. Cysts complicated by wide dermal adhesion, inflammation, or infection were excluded.

The surgical procedure was as follows: The skin overlying the site was cleaned with alcohol and normal saline. Under local anesthetic infiltration of $2 \%$ lidocaine with 1:100,000 epinephrine, a small hole having a diameter of $2 \mathrm{~mm}$ to $3 \mathrm{~mm}$ was made at the apex of the cyst using the $\mathrm{CO}_{2}$ laser. Most of the sebum inside of the cyst was extracted through the hole by gentle digital

Copyright (C) 2014 The Korean Society of Plastic and Reconstructive Surgeons

This is an Open Access article distributed under the terms of the Creative Commons Attribution Non-Commercial License (http://creativecommons.org/

licenses/by-nc/3.0/) which permits unrestricted non-commercial use, distribution, and reproduction in any medium, provided the original work is properly cited.

www.e-aps.org 


\section{Fig. 1. Schematic diagram of cyst excision}

(A) A small hole (diameter, 2-3 mm) was made at the apex of the cyst using the $\mathrm{CO}_{2}$ laser. $(\mathrm{B}, \mathrm{C})$ One leg of a mosquito hemostat was inserted into the cyst. Further, scraping was performed all around the cyst wall and on the base of the cyst. (D) Complete extraction of the cyst wall was achieved through the hole.

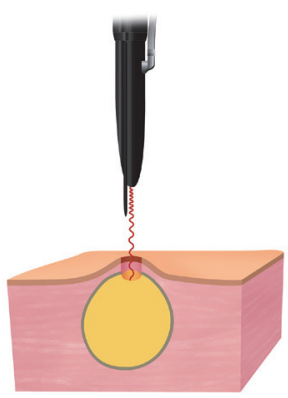

(A)

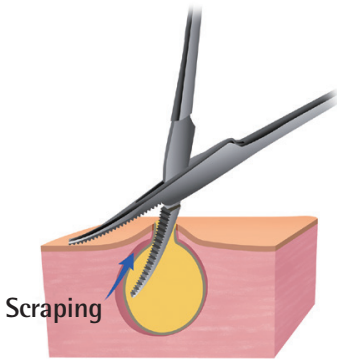

(B)

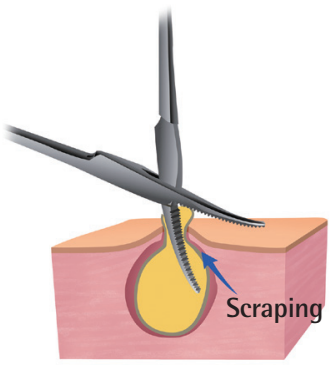

(C)

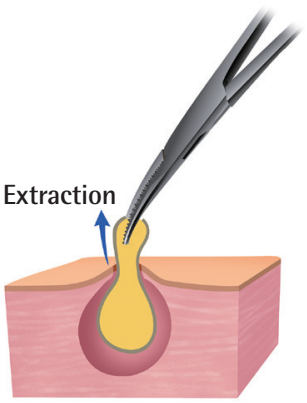

(D)

Fig. 2. A pinpoint scar left after laser hole excision

(A) A 47-year-old female with an epidermal cyst on her glabella. (B) Three months after laser hole excision.

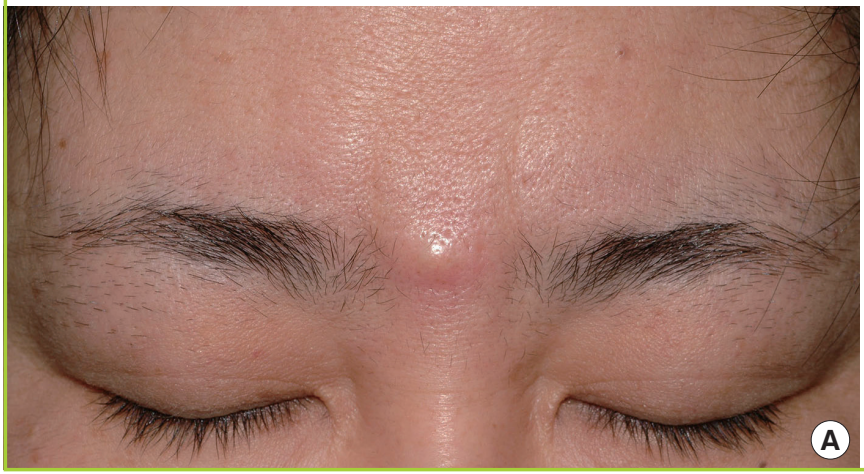

compression with gauze. One leg of a mosquito hemostat was inserted into the cyst, and the cyst wall was gently scraped away toward the hole using the horizontal grooves of the hemostat leg. While this scraping method was repeated all around the cyst wall, the cyst wall was gradually moved out through the hole and removed (Fig. 1). Following saline irrigation, the wound was inspected to ensure that the entire cyst wall had been removed. The skin hole was left when small in size but was closed with a stitch when large. A compression dressing was applied with ointment and gauze wetted with saline for five days, followed by simple dressing until complete healing was achieved.

The follow-up period was 3 to 5 months. Telephone interviews were conducted 6 months after the removal. One case on the cheek recurred, and the patient underwent the same procedure again. There was no further recurrence. Although the wounds occasionally resulted in mild erythema, they usually returned to normal skin color within 24 hours. The wounds were closed within 7 days. Further, a pinpoint scar similar to an acne scar was left in 21 cases and a small linear scar in 4 cases that required closure with a single stitch. Mild depression was observed in 3

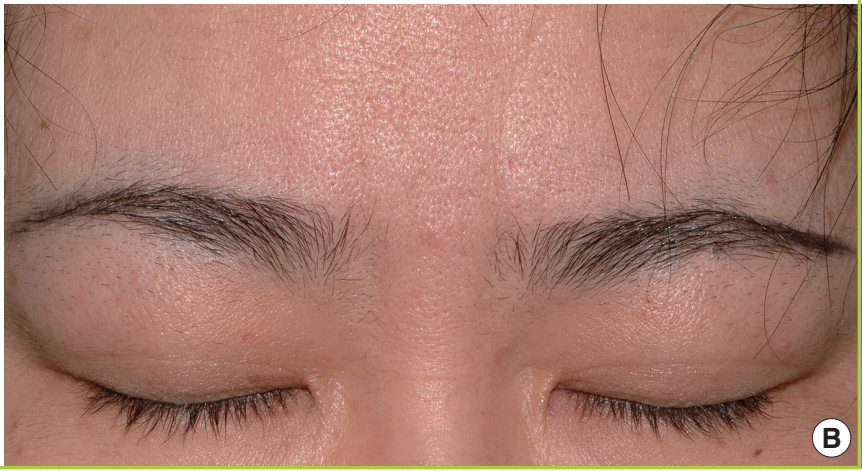

cases of neck cysts with skin thinness (Figs. 2, 3). There were no complications such as recurrence, infection, or hypertrophic scars. All of the patients were satisfied with the cosmetic results.

\section{DISCUSSION}

Epidermal cysts are common benign tumors of the skin that can appear anywhere on the body. Epidermal cysts on the face can be an aesthetic concern for patients, particularly since conventional management of an epidermal cyst involves a surgical procedure with unavoidable scarring. To prevent scarring associated with conventional surgical excision, several minimally invasive methods have been suggested, such as cyst removal through a minimal incision [2], cyst removal with a biopsy punch incision [3], and delayed excision of the cystic wall after sebum evacuation by laser punch [4].

One of the main causes of recurrence after excision of epidermal cysts is incomplete excision of the cystic wall. The body of an epidermal cyst underneath the skin loosely attaches to the subcutaneous tissue. Thus, the cystic wall of the body is easily 


\section{Fig. 3. A tiny linear scar with mild depression}

(A) A 31-year-old male with an epidermal cyst on his neck. (B) Four months after laser hole excision.
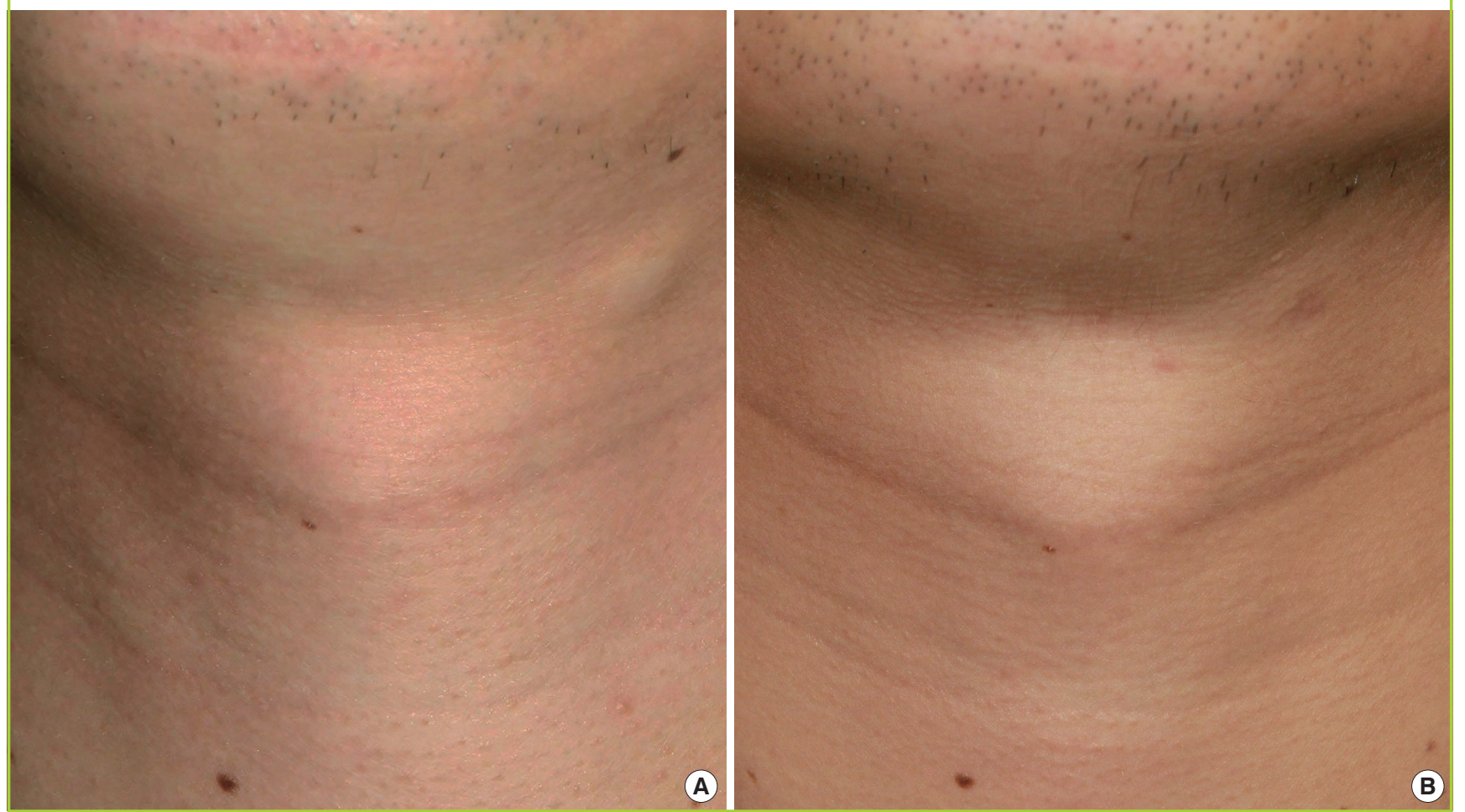

extracted by manual procedures if there has been no inflammation or fibrotic adhesion. However, the apex of epidermal cysts, including the secretory duct of the sebaceous gland is firmly attached to the dermis; thus, the epithelial lining of the apex is diffcult to remove by forceps extraction or by scraping. To remove it completely, the apex should be excised along with the attached dermis. In conventional excision techniques, the apex is excised in a fusiform shape. However, in the minimal incision technique [2], the apex is incised, not excised, which may preserve the epithelial lining of the apex and increase the risk of recurrence. Therefore, Yang and Yang [2] cauterized the underlying connective tissue with $20 \%$ trichloroacetic acid but did not describe the efficacy or the associated recurrence rate. As in the case of an excision, in our technique, the apex is completely ablated, including the surrounding dermis, by using a $\mathrm{CO}_{2}$ laser. The suspected reasons for our recurrence case are incomplete removal of the wide apex of the cyst or the uppermost portion of the body, which was firmly attached to the dermis, or duct obstruction of the surrounding sebaceous glands due to surgical injury during the procedure. We think that minimal incision techniques have a higher risk of recurrence than excision techniques because of the higher likelihood of incomplete removal of the epithelial lining of the apex.

A small skin incision or opening can limit the complete re- moval of a moderately sized or large cyst. In the 3-mm minimal incision technique [2], the treatment of 5 of the 6 patients who had a cyst with a diameter of $>1 \mathrm{~cm}$ failed and required an additional incision for cyst wall removal and sutures for wound closure. When our technique was used, the laser hole was 2 to $3 \mathrm{~mm}$ in diameter. Despite having the same diameter, a round hole provides a wider operating field than an incision and makes sebum extraction and cyst wall removal easier and more effective. This laser hole allows for excision of cysts that are $\leq 1.5 \mathrm{~cm}$ in diameter without additional procedures or delayed excision of the cyst wall.

Our laser hole technique is similar to a biopsy punch incision technique [3] with respect to the fact that the cyst is removed through a small hole. However, our technique has several advantages as compared to a biopsy punch hole. There is very little bleeding due to the laser coagulation of the hole wall, providing a clear visual operating field. It also induces delayed closure of the hole and enables prolonged fluid drainage, preventing infections and resulting in low recurrence. Therefore, this technique can be effectively used even for mild inflammatory cysts without skin adhesion.

A key aspect of our technique is laser ablation of the cyst apex and complete excision of the cyst wall using a scraping method with a mosquito hemostat. This technique is simple and results 
in minimal scarring, low recurrence rates, and high patient satisfaction. It may be a suitable minimally invasive method for removing epidermal cysts that are mobile, not inflamed, and $\leq 1.5$ $\mathrm{cm}$ in diameter.

\section{REFERENCES}

1. Zuber TJ. Minimal excision technique for epidermoid (sebaceous) cysts. Am Fam Physician 2002;65:1409-12.

2. Yang HJ, Yang KC. A new method for facial epidermoid cyst removal with minimal incision. J Eur Acad Dermatol Vene- reol 2009;23:887-90.

3. Mehrabi D, Leonhardt JM, Brodell RT. Removal of keratinous and pilar cysts with the punch incision technique: analysis of surgical outcomes. Dermatol Surg 2002;28:673-7.

4. Wu H, Wang S, Wu L, et al. A new procedure for treating a sebaceous cyst: removal of the cyst content with a laser punch and the cyst wall with a minimal postponed excision. Aesthetic Plast Surg 2009;33:597-9.

5. Nakamura M. Treating a sebaceous cyst: an incisional technique. Aesthetic Plast Surg 2001;25:52-6. 